\title{
Optimal Chiller Loading Using Modified Artificial Bee Colony Algorithm
}

\author{
Chang-Ming Lin, ${ }^{1,2 *}$ Ko-Ying Tseng, ${ }^{2}$ and Sheng-Fuu Lin ${ }^{1}$ \\ ${ }^{1}$ Institute of Electrical and Control Engineering, National Chiao Tung University, \\ No. 1001 University Road, Hsinchu 30010, Taiwan, R.O.C. \\ ${ }^{2}$ Energy and Environment Research Laboratories, Industrial Technology Research Institute, \\ Rm. 820, Bldg. 51, 8F, 195, Sec. 4, Chung Hsing Rd., Chutung, Hsinchu 31040, Taiwan, R.O.C.
}

(Received November 20, 2019; accepted April 25, 2020)

Keywords: optimal chiller loading, artificial bee colony algorithm, energy saving

In Taipei, over $45 \%$ of the energy used in buildings is for air-conditioning systems. In particular, multiple chiller systems consume about $70 \%$ of the energy in an air-conditioning system. Consequently, optimal chiller loading (OCL) or energy saving of a building is a vital issue. In this paper, we report a newly developed heuristic algorithm to solve OCL problems. A digital flow meter and a digital meter are installed to calculate the energy efficiency of a chiller. The exploration and exploitation of chiller loading can be efficiently improved without increasing the number of iterations by adopting the proposed modified artificial bee colony (MABC) algorithm. To demonstrate the performance of the proposed algorithm, it has been analyzed in comparison with other optimization methods. The result shows that the proposed algorithm can obtain a similar or better solution than previous algorithms. Therefore, it is a promising approach for solving the OCL problem.

\section{Introduction}

The electricity consumption of chillers in Taipei accounts for over $70 \%$ of total air-conditioning energy use. ${ }^{(1)}$ Multiple chillers commonly operate in parallel, wherein each chiller can either operate independently or serve as a backup so that stable standby freezing capacity and highly flexible maintenance scheduling in the field can be provided by controlling the operation of scheduling modes among the chillers. ${ }^{(1)}$ Since a multiple chiller system may be composed of different types of chillers with various characteristics, allocating the appropriate number of operating chillers and operation control points to ensure efficiency is a key to reducing the energy use of air-conditioning systems.

In recent years, there have been many studies globally on the optimal chiller loading (OCL) of multiple chiller systems. Chang proposed the branch and bound method, ${ }^{(2)}$ Lagrangian multiplier method, genetic algorithm (GA), and evolution strategy (ES) to solve the load optimization problem of multiple chiller systems. ${ }^{(3-5)}$ Ardakani et al. proposed the use of particle swarm optimization (PSO), an evolutionary algorithm based on the social behaviors of *Corresponding author: e-mail: linbarry@itri.org.tw https://doi.org/10.18494/SAM.2020.2812 
bird flocks, to solve the problem of OCL, and they proved that PSO is more efficient than binary GA and real GA in solving the problem of OCL. ${ }^{(6)}$ Lee et al. applied differential evolution (DE) to solve the problem of OCL. ${ }^{(7)}$ Coelho et al. proposed the use of the differential cuckoo search approach (DCSA) to improve the original cuckoo search approach (CSA), and obtained superior performance in OCL to that for GA, PSO, and DE. ${ }^{(8)}$ Recently, Zheng et al. have applied invasive weed optimization (IWO) and the theory of artificial fish swarm algorithms (AFSAs) to OCL with better results than those obtained with evolutionary theories such as PSO, DE, and ES. ${ }^{(9,10)}$

The artificial bee colony ( $\mathrm{ABC}$ ) algorithm is an evolutionary algorithm proposed by Karaboga in 2005, which simulates the honey-collecting behavior of bees. ${ }^{(11)}$ Compared with other evolutionary algorithms, the $\mathrm{ABC}$ algorithm has the advantages of a simpler concept and fewer control parameters, and it is superior in general numerical optimization problems. Although the ABC algorithm has many advantages, there are still some common problems with general evolutionary algorithms. When solving high-dimensional optimization problems, they are prone to falling into local optimal solutions, meaning that such algorithms lack global search capability. ${ }^{(12)}$

The function of a chiller is to move heat from one location to another. It is very common to use water or a water/glycol solution to transfer heat to and from the chiller, which may require the process chiller to have a reservoir and pumping system. An overview of a chiller system is shown in Fig. 1. The traditional chiller control method is based on constant flow control. A newer control method is to adjust the flow according to the chiller loading. Therefore, in this research, a digital flow meter and a digital meter are used to obtain the operating parameters of the chiller and calculate the chiller loading as the optimal control. In this paper, a modified artificial bee colony (MABC) algorithm is proposed to solve the problem of OCL. A newly developed bee colony self-information exchange mechanism is integrated into the $\mathrm{ABC}$ algorithm, which can effectively improve the algorithm by giving it improved search ability in its global diversity and local depth while also improving the execution efficiency and results of the global evolution method.

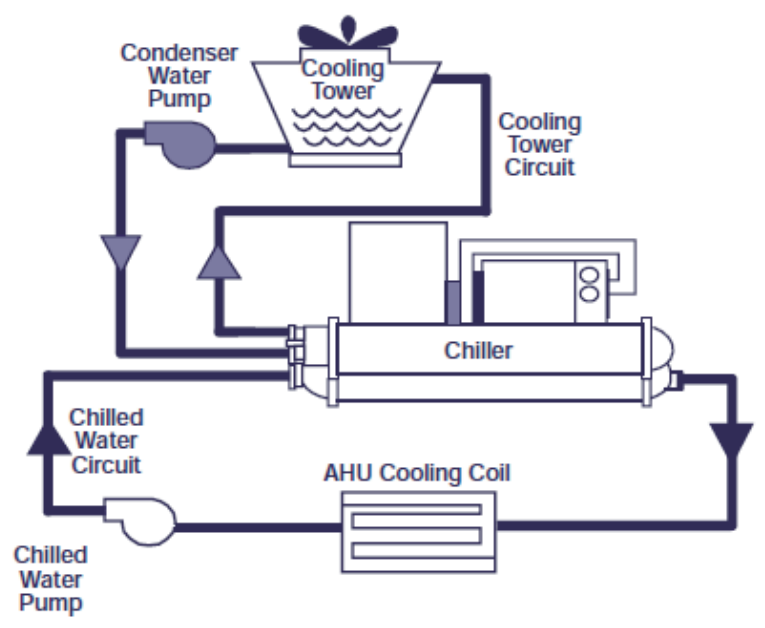

Fig. 1. Chiller operation. 
The structure of this paper is as follows. Section 2 describes in detail the problem of OCL, its target functions, and its constraints. Section 3 describes the evolution mechanism and characteristics of the MABC algorithm. Section 4 gives a comparison of sample results and Sect. 5 contains the conclusions.

\section{Multiple Chiller Systems}

Multiple chiller systems are widely used in the air-conditioning systems of many large buildings, which can provide greater operation flexibility than commonly used single chiller systems. A multiple chiller system is formed by connecting at least two chillers in parallel, wherein each chiller can independently supply a different refrigeration ton (RT) freezing capacity and operate efficiently according to different or similar performance curves of the chiller to provide diverse RT demand in the field. The architecture of a multiple chiller system is shown in Fig. 2.

Generally, the maximum peak load demand is taken as the maximum capacity in the design of chillers. However, owing to the relationship between the actual field demand and seasonal changes, the maximum peak load is usually only during the peak power consumption in summer, while most of the time the chiller operates under the partial load condition, resulting in excessive design volume and energy consumption. The partial load rate (PLR) relationship of a chiller can be expressed as

$$
P L R=\frac{\text { Chiller load }}{\text { Chiller rated load }}
$$

The power consumption of a chiller is related to PLR to a certain extent. The power consumption equations of the chillers established in Ref. 8 are given by Eqs. (2) and (3).

$$
P_{i}=a_{i}+b_{i} \times P L R_{i}+c_{i} \times P L R_{i}^{2}
$$

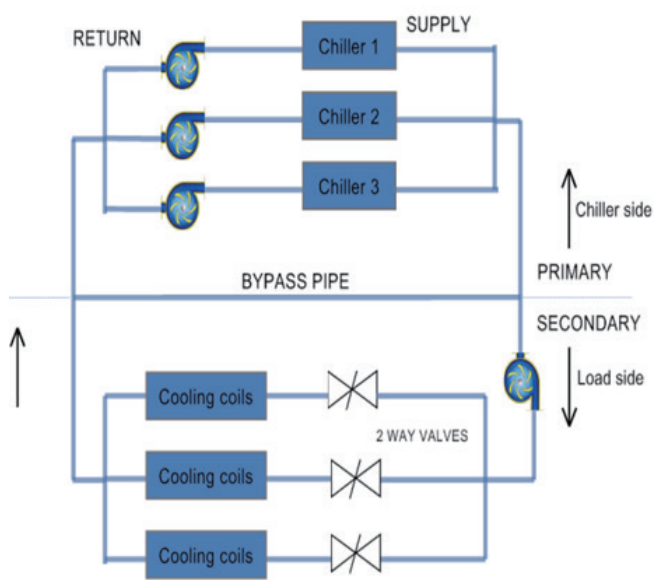

Fig. 2. (Color online) Architecture of a multiple chiller system. 
Equation (2) is used in the power consumption model of the example chiller. The coefficients $a_{i}, b_{i}$, and $c_{i}$ in the equation define the relationship between the power consumption and PLR of each chiller in the analysis example, similarly to in the literature. ${ }^{(8)}$ The upper and lower limits of the PLR range from 0.0 to 1.0 .

The objective of the overall OCL optimization is to find the partial load of each chiller in the multiple chiller system according to the demand of the field freezing load to minimize power consumption. Therefore, the target function for OCL optimization is defined as

$$
\operatorname{Obj}_{\text {Function }}=\text { Minimize } \sum_{i=1}^{n} P_{i}
$$

where parameter $i$ refers to the $i$ th chiller, $n$ refers to the total number of chillers in the whole multiple chiller system, and $P_{i}$ refers to the power consumption (unit: $\mathrm{kW}$ ) of the $i$ th chiller. The objective of the equation is to find the lowest possible total power consumption of the multiple chiller system.

The OCL problem is solved with two constraints. ${ }^{(8)}$ The first constraint is that the total output value of frozen tons must equal the demand of frozen tons in the field, which is expressed as

$$
\sum_{i=1}^{n} P L R_{i} \times Q_{i}=C L
$$

where $Q_{i}$ refers to the rated RT capacity of the $i$ th chiller and $C L$ is the total number of RTs required in the field.

The second restriction is that the PLR of each chiller cannot be lower than $30 \%$, ${ }^{(8)}$ which is expressed as

$$
P L R_{i} \geq 0.3 .
$$

\section{MABC Algorithm}

The purpose of developing the MABC algorithm is to improve the search efficiency and diversity of the optimal solution of the original $\mathrm{ABC}$ algorithm. However, an optimization algorithm with high convergence speed usually has the disadvantage of premature convergence. In contrast, if the algorithm has a high search diversity, it needs more iterations to reach the optimal solution with relatively high accuracy. ${ }^{(13-15)}$

\subsection{ABC algorithm}

The $\mathrm{ABC}$ algorithm is an evolutionary algorithm that simulates the honey-collecting behavior of bees. ${ }^{(1)}$ There are three different types of bees in the overall calculation mode and three different characteristics of bees: employed bees, onlooker bees, and scout bees. Employed 
bees are responsible for searching for nectar sources, onlooker bees search for nectar sources according to information provided by employed bees, and scout bees begin random searches when the food sources have not been updated for a period of time.

Executing the algorithm begins with setting the parameters required by the bee colony algorithm, including the number of employed bees (SN), the maximum number of iterations (MaxGen), the cumulative number of unimproved times (limit), and randomly generated food sources within the design range. The best solution is searched for through the employed bee searching mechanism. The food sources found by the employed bees are then sent to the onlooker bees for collecting. If a certain food source cannot be updated after repeated iterations, the food source will be abandoned and the scout bee detecting mechanism will be started to restart random searching. The whole algorithm repeats itself until the MaxGen is reached.

\subsubsection{Food source renewal mechanism of employed bees}

A location randomly generated in the solution space is adopted for each employed bee, and the cost value of each food source location needs to be calculated. The formula for searching the food source location of each employed bee is

$$
V_{i, G+1}=X_{i, G}+r 1 \times\left(X_{i, G}-X_{k, G}\right) .
$$

$V_{i, G+1}$ is the new food source selected by the employed bee, $X_{i, G}$ is the original food source where the employed bee is located, $X_{k, G}$ is the food source where another randomly selected employed bee is located, and $r 1$ is a real value randomly set from -1.0 to 1.0 according to the reference variable. ${ }^{(11)}$ After the location of the new food source is calculated, the cost values of the two food source locations $V_{i, G+1}$ and $X_{i, G}$ are compared. If the cost value of the new food source is higher than that of the original food source, the new food source is selected; otherwise, the location of the original food source is retained.

\subsubsection{Food source renewal mechanism of onlooker bees}

After the food source locations of all employed bees are updated through the mechanism in Sect. 3.1.1, the rotating method is used to select the food source locations searched by the employed bees as the initial searching locations of onlooker bees. The calculation formula of the rotating method is

$$
P_{i, j}=\frac{f i t_{i}}{\sum_{n=1}^{S N} f i t_{n}},
$$

where $P_{i}$ is the probability that the $i$ th food source location is selected and $f i t_{i}$ is the cost value calculated for the $i$ th food source location. The rotating method is performed by using $P_{i j}$ calculated by Eq. (7) to generate the location where the onlooker bee searches for new food 
sources, which is calculated by Eq. (6). After the onlooker bee calculates the new food source location, the cost values of the new and original food source locations are compared, and the location with the higher cost value will be selected for the onlooker bee.

\subsubsection{Food source renewal mechanism of scout bees}

When any employed bee's food source location has not been updated continuously for more than the number of iterations in the initially set limit, the food source renewal mechanism of scout bees restarts. These bees abandon the food source location and randomly generate a new food source location in the design space for the employed bee.

\subsection{MABC algorithm}

The original $\mathrm{ABC}$ algorithm has good convergence characteristics in the calculation strategy, but with regard to search breadth, it only relies on the mechanism of scout bees for continuously unimproved food source locations. Hence, it is easy for the algorithm to fall into a regional optimal solution when searching for multiple variables to optimize the problem. Therefore, in this study, we developed the MABC, which includes two new mechanisms to improve the search breadth and speed of multiple variables for optimization of the problem.

\subsubsection{Updated mechanism of optimal food source location}

The food source updating mechanism of the traditional ABC algorithm, such as Eq. (6), uses another randomly selected food source location for subtraction from its own food source location for calculation. The search speed for the best solution of Eq. (6) is low and close to that of a random search. If the selected food source location is far from the global best solution, the calculated cost value of the new food source location may not be improved. In this study, we take the mutation mechanism of the $\mathrm{DE}^{(16-19)}$ method and modify it by replacing the original Eq. (6) to improve the search speed of the ABC algorithm. The vector search mechanism of the original DE method is expressed by

$$
V_{i, G+1}=X_{\text {best }, G}+F \times\left(X_{i, G}-X_{k, G}\right) .
$$

In the mutation formula of the DE method, different individuals $X_{i, j}$ and $X_{k, j}$ are randomly selected from individuals 1 to $P$ in the population, which are subtracted to obtain a vector difference, multiplied by the weight value $F$, and finally added to the best target vector $X_{\text {best }, j}$ in the current generation to obtain the interference vector $V_{i, G+1}$ of the next generation. In this newly proposed mechanism, the food source searching mechanism is expressed by

$$
V_{i, G+1}=X_{\text {best }, G}+r 1 \times\left(X_{i, G}-X_{k, G}\right) .
$$


Here, $X_{\text {best }, G}$ is the food source location with the highest cost value searched for by all employed bees or onlooker bees at present, $V_{i, G+1}$ is the new food source searched for by the employed bee, $X_{i, G}$ is the original food source where the employed bee is located, $X_{k, G}$ is the food source location of another randomly selected employed bee, and $r 1$ is a random real value between -1.0 and 1.0. Equation (9) is used to replace Eq. (7) of the ABC algorithm to search for new food source locations, greatly improving the convergence speed of the algorithm.

\subsubsection{Information exchange mechanism of onlooker bees}

The search-diversifying mechanism of the original $\mathrm{ABC}$ algorithm has no other way to improve the search diversity of the algorithm except to randomly generate new locations for food source locations where the cost values have not been improved for several iterations. To solve this problem, we propose a new information exchange mechanism for onlooker bees. After the implementation of the mechanism for onlooker bees, new food source information generated from onlooker bees or original employed bees is adopted to improve the search diversity for the optimal solution. This is expressed by

$$
\begin{array}{ll}
U_{j i, G+1}=V_{j i, G+1}, & \text { if } R \leq R_{-} \text {set } \\
U_{j i, G+1}=X_{j i, G}, & \text { if } R>R_{-} \text {set } \\
j=1,2, \ldots, D, &
\end{array}
$$

where $D$ is the total number of design variables at the food source location of the onlooker bee, $V_{j i, G+1}$ is the $j$ th variable at the food source location of the onlooker bee calculated through Eq. (9), $X_{j i, G}$ is the $j$ th variable at the food source location of the employed bee originally selected through the rotating method, and $U_{j i, G+1}$ is the $j$ th variable value at the new onlooker bee food source calculated through Eq. (10). $R$ is a random real value between 0.0 and 1.0 and is equal to the information exchange probability of the onlooker bee. Through the information exchange mechanism for onlooker bees, the search breadth of the $\mathrm{ABC}$ algorithm can be effectively improved. The overall architecture of the MABC algorithm is shown in Fig. 3.

\section{Results and Discussion}

This example is a multiple chiller system composed of six chillers. The purpose is to calculate the combination of the six chillers giving minimum power consumption for different RT requirements. The analysis example has five different field RT requirements: 6858 (90\%), $6477(85 \%), 6096(80 \%), 5717(75 \%)$, and $5334(70 \%)$. The power consumption of the chillers in analysis example 1 is given by Eq. (2). The rated RTs and power consumption parameters $\left(a_{i}, b_{i}, c_{i}\right)$ that each chiller can provide are shown in Table 1, and Table 2 shows a comparison of the optimization results of the search in this study with those in the literature. ${ }^{(6,8,9,20)}$

Figure 4 is a convergence performance chart of the MABC algorithm toward the optimal solution for different refrigeration requirements. The optimal solution for all different studied 


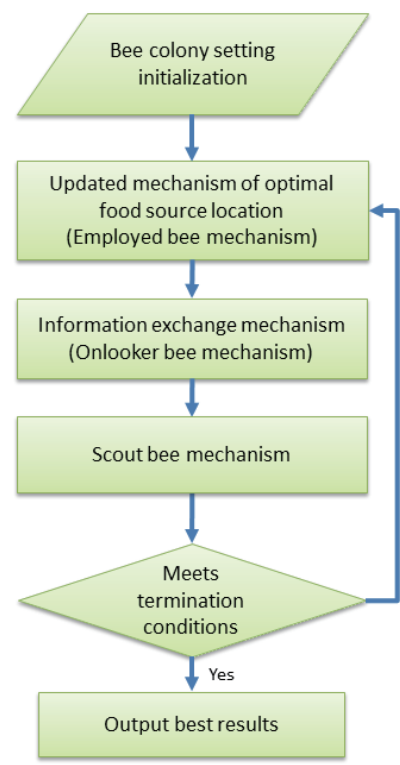

Table 1

Power consumption coefficient and rated RT information in analysis example.

\begin{tabular}{lcrrc}
\hline Chiller & \multicolumn{1}{c}{$a_{i}$} & \multicolumn{1}{c}{$b_{i}$} & \multicolumn{1}{c}{$c_{i}$} & Rated $R T$ \\
\hline 1 & 399.345 & -122.12 & 206.30 & 1280 \\
2 & 287.116 & 80.04 & 700.48 & 1280 \\
3 & -120.505 & 1525.99 & -502.14 & 1280 \\
4 & -19.121 & 898.76 & -98.15 & 1280 \\
5 & -95.029 & 1202.39 & -352.16 & 1280 \\
6 & 191.75 & 224.86 & 524.04 & 1280 \\
\hline
\end{tabular}

Fig. 3. (Color online) Architecture of MABC algorithm.

Table 2

Comparison of the results of the method in this study with those in the literature.

\begin{tabular}{|c|c|c|c|c|c|c|c|c|c|c|c|}
\hline \multirow[t]{2}{*}{ CL } & $\begin{array}{l}\text { Chiller } \\
\text { number }\end{array}$ & $\mathrm{SA}^{(20)}$ & $\begin{array}{l}\text { Power } \\
(\mathrm{kW})\end{array}$ & $\mathrm{PSO}^{(6)}$ & $\begin{array}{l}\text { Power } \\
(\mathrm{kW})\end{array}$ & $\operatorname{DCSA}^{(8)}$ & $\begin{array}{l}\text { Power } \\
(\mathrm{kW})\end{array}$ & $\mathrm{IWO}^{(9)}$ & $\begin{array}{l}\text { Power } \\
(\mathrm{kW})\end{array}$ & $\begin{array}{c}\text { Modified } \\
\text { ABC }\end{array}$ & $\begin{array}{l}\text { Power } \\
(\mathrm{kW})\end{array}$ \\
\hline & $i$ & $P L R_{i}$ & & $P L R_{i}$ & & $P L R_{i}$ & & $P L R_{i}$ & & $P L R_{i}$ & \\
\hline \multirow{5}{*}{$\begin{array}{l}6858 \\
(90 \%)\end{array}$} & 1 & 0.7789 & \multirow{5}{*}{4777.03} & 0.8026 & \multirow{5}{*}{4739.53} & 0.812726 & \multirow{5}{*}{4738.575} & 0.8127 & & 0.81263 & \multirow{5}{*}{4738.575} \\
\hline & 2 & 0.7587 & & 0.7799 & & 0.749619 & & 0.7492 & & 0.749632 & \\
\hline & 3 & 0.9791 & & 0.9996 & & 1.000000 & & 1.000000 & $4738 \quad 575$ & 1.000000 & \\
\hline & 4 & 0.9781 & & 0.9998 & & 1.000000 & & 1.000000 & $4 / 50.515$ & 1.000000 & \\
\hline & 6 & 0.9265 & & 0.8183 & & 0.838559 & & 0.8390 & & 0.838642 & \\
\hline \multirow{4}{*}{$\begin{array}{l}6477 \\
(85 \%)\end{array}$} & 1 & 0.8051 & \multirow{4}{*}{4453.67} & 0.7606 & \multirow{4}{*}{4423.04} & 0.727731 & \multirow{4}{*}{4421.649} & 0.7275 & & 0.727536 & \multirow{4}{*}{4421.649} \\
\hline & 2 & 0.6056 & & 0.6555 & & 0.656132 & & 0.6563 & & 0.657360 & \\
\hline & 3 & 0.9689 & & 1.0000 & & 1.000000 & & 1.000000 & 4421640 & 1.000000 & \\
\hline & 4 & 0.9941 & & 1.0000 & & 1.000000 & & 1.000000 & 4421.049 & 1.000000 & \\
\hline \multirow{5}{*}{$\begin{array}{l}6096 \\
(80 \%)\end{array}$} & 2 & 0.5743 & \multirow{5}{*}{4178.73} & 0.5798 & \multirow{5}{*}{4147.69} & 0.562645 & \multirow{5}{*}{4143.706} & 0.5628 & & 0.562221 & \multirow{5}{*}{4143.706} \\
\hline & 3 & 0.9675 & & 0.9991 & & 1.000000 & & 1.000000 & 14270 & 0.999999 & \\
\hline & 4 & 0.9798 & & 0.9979 & & 1.000000 & & 1.000000 & $143 . / 06$ & 0.999999 & \\
\hline & 5 & 0.9845 & & 0.9921 & & 1.000000 & & 1.000000 & & 0.999999 & \\
\hline & 6 & 0.7338 & & 0.5710 & & 0.594490 & & 0.5944 & & 0.594166 & \\
\hline \multirow{5}{*}{$\begin{array}{l}5717 \\
(75 \%)\end{array}$} & 1 & 0.6140 & \multirow{5}{*}{3925.51} & 0.7713 & \multirow{5}{*}{3921.07} & 0.843697 & \multirow{5}{*}{3840.055} & 0.0000 & & 0.843108 & \multirow{5}{*}{3838.215} \\
\hline & 2 & 0.4429 & & 0.7177 & & 0.783794 & & 0.7151 & & 0.783339 & \\
\hline & 3 & 0.9891 & & 0.3000 & & 0.000001 & & 1.000 & 3842552 & 0.000000 & \\
\hline & 4 & 0.8867 & & 0.9991 & & 1.000000 & & 1.000 & 3842.553 & 0.999999 & \\
\hline & 5 & 0.9841 & & 1.0000 & & 1.000000 & & 1.000 & & 0.999999 & \\
\hline
\end{tabular}




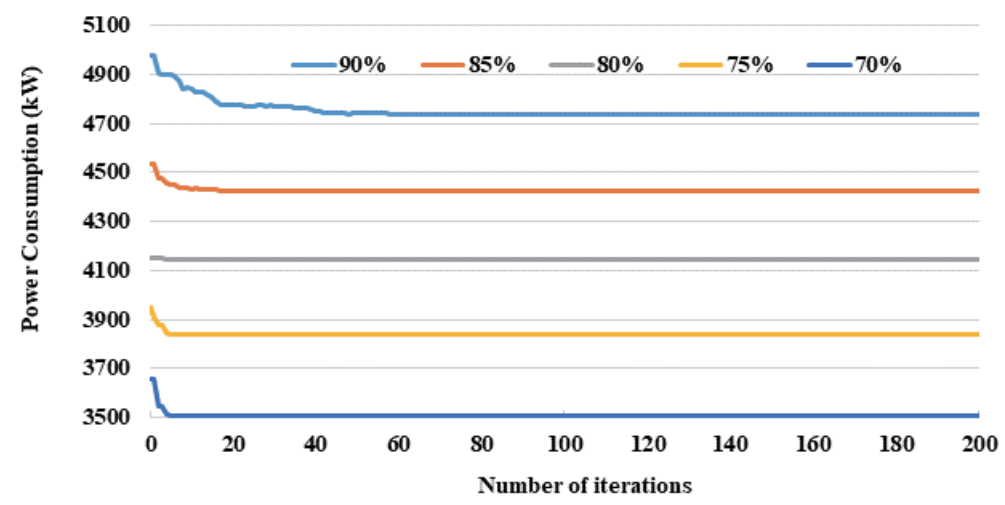

Fig. 4. (Color online) Comparison of convergence speed of the MABC algorithm.

cases was found by carrying out 60 iterations, while the lowest power consumption was obtained by carrying out 20 iterations for the four results with field RT requirements of 6477 (85\%), $6096(80 \%), 5717(75 \%)$, and $5334(70 \%)$, proving that the method proposed in this study can effectively solve the OCL problem.

From the results in Fig. 4, it can be seen that for the field RT requirements of $6858(90 \%)$, $6477(85 \%), 6096(80 \%)$, and $5334(70 \%)$, similar power consumption results to those in the literature can be obtained. For the field RT requirement of 5717 (75\%), this method can find better chiller power consumption results than those in the literature, proving that the MABC algorithm is suitable for solving the load optimization problem of multiple chillers.

\section{Conclusions}

The MABC algorithm proposed in this paper integrates new evolution mechanisms with two different characteristics of convergence speed and search diversity, simultaneously improving the breadth and convergence speed of the optimal solution. In this study, we install a flow meter on the pipeline to calculate the load of the chiller and use this value to determine the efficiency of the chiller. According to the results of the analysis example and other literature, the MABC algorithm is suitable for optimizing the minimum power consumption combination of multiple chiller systems. Not only can solutions close to or even better than those in the literature be obtained, but also the convergence speed when searching for the best solution is also extremely high. The results confirm that the MABC algorithm has better performance and stability than other methods and can be applied to similar optimization problems. In the future, this research technology can be combined with some software platforms, using information and communication technology and Internet of Things technology to expand the application of energy saving. ${ }^{(21,22)}$

\section{Acknowledgments}

We thank National Chiao Tung University and Tatung University for their generous provision of computing time. This research was funded by the Bureau of Energy, Ministry of Economic Affairs, R.O.C. (Taiwan). 


\section{References}

1 M. Schwedler and A. Yates: Multiple-chiller-system Design and Control, Trane (2001).

2 Y. C. Chang: Electr. Pow. Syst. Res. 69 (2004) 221. https://doi.org/10.1016/j.epsr.2003.10.012

3 Y. C. Chang, L. Fu-Ann, and L. C. Huang: Energ. Convers. Manage. 46 (2005) 2158. https://doi.org/10.1016/ j.enconman.2004.10.012

4 Y. C. Chang: Appl. Therm. Eng. 25 (2005) 2800. https://doi.org/10.1016/j.applthermaleng.2005.02.010

5 Y. C. Chang: Energ. Buildings 39 (2007) 437. https://doi.org/10.1016/j.enbuild.2005.12.009

6 A. J. Ardakani, F. F. Ardakani, and S. H. Hosseinian: Energ. Buildings 40 (2008) 2177. https://doi.org/10.1016/ j.enbuild.2008.06.010

7 W. S. Lee, Y. T. Chen, and Y. Kao: Energ. Buildings 43 (2011) 599. https://doi.org/10.1016/j.enbuild.2010.10.028

8 L. D. S. Coelho, C. E. Klein, S. L. Sabat, and V. C. Mariani: Energy 75 (2014) 237. https://doi.org/10.1016/ j.energy.2014.07.060

9 Z. X. Zheng and J. Q. Li: Energ. Buildings 161 (2018) 80. https://doi.org/10.1016/j.enbuild.2017.12.020

10 Z. X. Zheng, J. Q. Li, and P. Y. Duan: Math. Comput. Simulat. 155 (2019) 227. https://doi.org/10.1016/ j.matcom.2018.04.013

11 D. Karaboga: An Idea Based on Honey Bee Colony for Numerical Optimization (Technical Report-TR06, 2005).

12 G. Zhu and S. Kwong: Appl. Math Comput. 217 (2010) 3166. https://doi.org/10.1016/j.amc.2010.08.049

13 K. C. Tan, S. C. Chiam, A. A. Mamun, and C. K. Goh: Eur. J. Oper. Res. 197 (2009) 701. https://doi. org/10.1016/j.ejor.2008.07.025

14 K. J. Binkley and M. Hagiwara: Trans. Jpn. Soc. Artif. Intell. 23 (2008) 27. https://doi.org/10.1527/tjsai.23.27

15 M. G. Epitropakis, V. P. Plagianakos, and M. N. Vrahatis: IEEE C. Evol. Computat. (2008). https://doi. org/10.1109/CEC.2008.4631159

16 R. Storn and K. Price: J. Global. Optim. 11 (1997) 341. https://doi.org/10.1023/A:1008202821328

17 C. Y. Wu and K. Y. Tseng: Struct. Multidiscip. Optim. 42 (2010) 939. https://doi.org/10.1007/s00158-010-05239

18 C. Y. Wu and K. Y. Tseng: J. Mech. Eng. Sci. 224 (2010) 443. https://doi.org/10.1243/09544062JMES1764

19 C. Y. Wu and K. Y. Tseng: 2010 3rd Int. Joint Conf. Computational Science and Optimization (IEEE 2010) 501. https://doi.org/10.1109/CSO.2010.185

20 Y. C. Chang: Energy 31 (2006) 1883. https://doi.org/10.1016/j.energy.2005.10.018

21 A. D. Kin-Tak Lam, S. D. Prior, S. T. Shen, S. J. Young, and L. W. Ji: 2019 Engineering Innovation and Design (CRC Press, London). https://doi.org/10.1201/9780429019777

22 A. D. Kin-Tak Lam, S. D. Prior, S. T. Shen, S. J. Young, and L. W. Ji: 2020 Smart Science, Design \& Technology (CRC Press, London). https://doi.org/10.1201/9780429058127

\section{About the Authors}

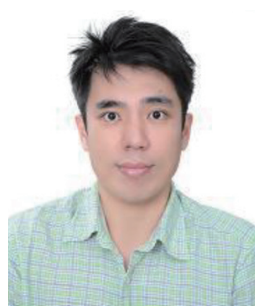

Chang-Ming Lin was born in Tainan, R.O.C., in 1980. He received his B.S. degree in mechanical engineering from National Yunlin University of Science \& Technology in 2003 and his M.S. degree in mechanical engineering from Widener University in 2007. He is currently an engineer at the Industrial Technology Research Institute (ITRI). His current research interests include data mining and energy-saving control. (linbarry@itri.org.tw)

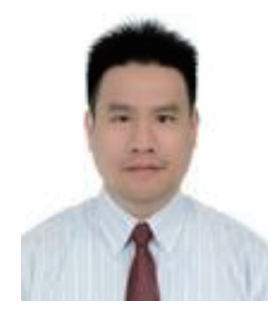

Ko-Ying Tseng was born in 1978. He received his M.S. and Ph.D. degrees in mechanical engineering in 2003 and 2010, respectively, from Tatung University. He is currently an engineer at the Green Energy and Environment Research Lab., ITRI. His research interests are engineering optimization using bio-inspired algorithms and artificial intelligence. (optbilly@gmail.com) 
Sheng-Fuu Lin was born in Tainan, R.O.C., in 1954. He received his B.S. and M.S. degrees in mathematics from National Taiwan Normal University in 1976 and 1979, respectively, his M.S. degree in computer science from the University of Maryland in 1985, and his Ph.D. degree in electrical engineering from the University of Illinois, Champaign, in 1988. Since 1988, he has been on the Faculty of the Department of Electrical and Control Engineering at National Chiao Tung University, Hsinchu, Taiwan, where he is currently a professor. His research interests include image processing, pattern recognition, fuzzy theory, and automatic target recognition.

(sflin@mail.nctu.edu.tw) 